\section{Die Beiträge von direkter und indirekt diffuser Sonnenstrahlung im UV-Spektrum}

\section{Stick}

Institut für Medizinische Klimatologie der Christian-AlbrechtsUniversität zu Kiel (Direktor: Prof. Dr. C. Stick)
Zusammenfassung. Die Ultraviolettstrahlung unterscheidet sich in mehrerer Hinsicht von den anderen Anteilen des Sonnenspektrums. Ziel dieser Arbeit ist es, anhand spektroradiometrischer Messungen darzustellen, wie und in welchem Ausmaß die indirekt-diffuse und direkte Strahlung bei verschiedenen Wellenlängen des UV-Bereichs in Abhängigkeit vom Sonnenhöhenwinkel variieren. Die Messungen wurden auf der Nordseeinsel Sylt auf $54^{\circ} 55,5^{`} \mathrm{~N}, 8^{\circ} 18,6^{`} \mathrm{O}$ durchgeführt. Die UV-Spektren wurden an einem klaren, wolkenlosen Sommertag um die Sonnenwende mit einem Doppelmonochromator-Radiometersystem (DM 150, Bentham Comp., Reading, England) aufgenommen. Ein Teflon-Diffuser mit angenäherter Kosinus-Empfangscharakteristik diente als Eingangsoptik des Spektroradiometers. Für die Messung der direkten Sonnenstrahlung wurde der Diffusor so ausgerichtet, dass die Sonnenstrahlen senkrecht auf ihn trafen. Eine speziell nach den Richtlinien der WMO für die Messung der direkten Sonnenstrahlung mit Pyrheliometern (Öffnungshalbwinkel $2,5^{\circ}$, Böschungswinkel $1^{\circ}$ ) angefertigte Blende sorgte dafür, dass ausschließlich die Bestrahlungsstärke der direkten Sonnenstrahlen gemessen wurde. Umgekehrt wurde die indirekt-diffuse Strahlung mit dem horizontal ausgerichteten Diffusor gemessen, der mit einer Schattenscheibe von der direkten Sonnenstrahlung verschattet wurde. Um die beiden Messungen vergleichen zu können, wurden die Werte der direkten Strahlung bezogen auf die horizontale Fläche umgerechnet. Aus den Spektren wurden die folgenden sechs Wellenlängen analysiert: $305 \mathrm{~nm}$ und $310 \mathrm{~nm}$ aus dem UVB-, bzw. $330 \mathrm{~nm}$, $340 \mathrm{~nm}, 360 \mathrm{~nm}$ und $380 \mathrm{~nm}$ aus dem UVA-Bereich. Naturgemäß nehmen sowohl die direkte als auch die indirekte Strahlung mit der Sonnenhöhe zu. Doch tun sie dies in unterschiedlicher Weise. Bei geringen Sonnenhöhen etwa bis zum Sonnenhöhenwinkel von $30^{\circ}$ überwiegt bei allen Wellenlängen des UV-Bereichs die indirekte Strahlung. Bei $305 \mathrm{~nm}$ und $310 \mathrm{~nm}$ trifft das sogar bis zur Sonnenhöhe von fast $60^{\circ}$ am Mittag zu. Bei diesen Wellenlängen liefert die diffuse Strahlung bei der Sonnenhöhe von $30^{\circ}$ fast $80 \%$ der Globalstrahlung und noch rund $56 \%$ bei $60^{\circ}$ Sonnenhöhe. Dies bedeutet, dass die indirekte Strahlung die direkte während des ganzen Tages übertrifft. Dieses Verhältnis ändert sich im UVA-Bereich: Je länger die Wellenlängen werden und je höher die Sonne steigt, umso gewichtiger wird die direkte Strahlung. Bei $330 \mathrm{~nm}$ sind die Bestrahlungsstärken der

Akt Dermatol 2001; 27: 7-12

(c) Georg Thieme Verlag Stuttgart $\cdot$ New York ISSN 0340-2541 direkten und indirekten Strahlung bei $60^{\circ}$ Sonnenhöhe etwa gleich groß. Bei $340 \mathrm{~nm}$ verschiebt sich dieses Gleichgewicht zu etw $50^{\circ}$, bei $360 \mathrm{~nm} \mathrm{zu} 40^{\circ}$ und bei $380 \mathrm{~nm}$ zu ungefähr $30^{\circ}$. Insgesamt lässt sich feststellen: je kürzer die Wellenlänge, umso mehr wird die UV-Strahlung als diffuse indirekt aus dem Himmelsblau gestreute Strahlung eingestrahlt. Dies ist deswegen von besonderem Interesse, weil die kurzen Wellenlängen genau diejenigen sind, welche auf die Haut am stärksten erythemerzeugend wirken.

The Contributions of Direct and Indirect Diffuse Solar Radiation in the UV-Spectrum. Ultraviolet radiation differs in some respects from other parts of the solar spectrum. Spectroradiometric measurements were used to demonstrate how and to which extent direct and indirect diffuse radiation vary at certain wavelengths in the UV range depending on the solar elevation, i.e. during the course of a day. Measurements were done on the North Sea island of Sylt at $54^{\circ} 55.5^{`} \mathrm{~N}, 8^{\circ} 18.6^{`} \mathrm{E}$. UVspectra were measured during a clear cloudless summer day using a double monochromator system (DM 150, Bentham Comp., Reading, England). A Teflon diffuser with an approximate cosine response served as input optic of the spectroradiometer. Direct solar radiation was measured when the receiving surface of the diffuser was arranged to be normal to the incident sun rays. A custom made aperture constructed according to the WMO guidelines for measurement of direct solar radiation by pyrheliometers (opening half-angle $2.5^{\circ}$, slope angle $1^{\circ}$ ) ensured that only the irradiance of the direct sun rays was measured. Conversely the diffuse radiation was measured when the horizontally adjusted diffuser was shaded from the direct sun rays by a shadow disk. To make both measurements comparable, the measured irradiance of the direct radiation was converted in relation to a horizontal plane. Out of the spectra the following six wavelengths were analyzed: $305 \mathrm{~nm}$ and $310 \mathrm{~nm}$ in the UVB and $330,340,360$, and $380 \mathrm{~nm}$ in the UVA, respectively. Naturally both direct and indirect radiation increase with the elevation of the sun. However, they do so in a different manner. At low elevation up to about $30^{\circ}$ indirect radiation prevails at all wavelengths in the UV range. At $305 \mathrm{~nm}$ and $310 \mathrm{~nm}$ this holds true even up to a solar elevation of almost $60^{\circ}$ at noon. At these wavelengths indirect radiation contributes almost $80 \%$ to the global irradiance at $30^{\circ}$ solar elevation and still about $56 \%$ at $60^{\circ}$. This means that indirect radiation exceeds direct radiation during the entire day. This relationship changes in the UVA range. The longer the wavelengths and the higher the sun the more im- 
portant becomes the direct radiation. At $330 \mathrm{~nm}$ the irradiances of direct and indirect radiation are roughly equal at a solar elevation of $60^{\circ}$. At $340 \mathrm{~nm}$ this point of even shifts to about $50^{\circ}$, at $360 \mathrm{~nm}$ to $40^{\circ}$, and at $380 \mathrm{~nm}$ to roughly $30^{\circ}$. All in all, the shorter the wavelength the more the UV radiation is irradiated as diffuse indirect radiation scattered from the blue of the sky. This is of particular interest because the short wavelengths are precisely those which cause the most skin erythema.

\section{Einleitung}

Der Spektralbereich der ultravioletten Sonnenstrahlung weist eine Reihe von Besonderheiten gegenüber dem sichtbaren Licht und der Infrarotstrahlung der Sonne auf. Für den Dermatologen am bedeutendsten ist zuerst die ungewöhnlich starke biologische Wirksamkeit der UV-Strahlung auf die Haut [1]. Diese wird einerseits zu therapeutischen Zwecken genutzt [2], andererseits ist sie jedoch auch für lichtbedingte Schäden an der Haut und am Auge verantwortlich [3].

Sodann zeichnet sich die solare UV-Strahlung dadurch aus, dass ihre Bestrahlungsstärke sehr viel stärker vom Sonnenhöhenwinkel abhängt als die der anderen Spektralbereiche. Hochauflösende Spektralradiometer erlauben es, die ultraviolette Sonnenstrahlung nach Wellenlängen geordnet in Nanometerschritten zu zerlegen und die Bestrahlungsstärke jeder einzelnen Wellenlänge zu bestimmen. Aus solchen Messungen wird deutlich, dass sich die Zusammensetzung der ultravioletten Sonnenstrahlung während eines Tages permanent ändert und die Bestrahlungsstärken der verschiedenen Wellenlängen selbst innerhalb des UV-Spektralbereichs in sehr unterschiedlicher Weise vom Sonnenhöhenwinkel abhängen [4]. Speziell im kurzwelligen UVB-Bereich ist diese Abhängigkeit besonders stark.

Eine weitere Eigenschaft dieser Strahlung am kurzwelligen Ende des Sonnenspektrums ist ihre ungewöhnlich starke Streuung in der Atmosphäre. Die folgende Arbeit ist der Darstellung dieser Eigenschaft der ultravioletten Sonnenstrahlung gewidmet. Die Kenntnis auch dieser speziellen Eigenart der kurzwelligen UV-Strahlung gewinnt angesichts dramatisch steigender Inzidenzen der strahlungsbedingten Hautkrebsarten und anderer durch die Sonnenstrahlung hervorgerufenen Hautschäden zunehmende Bedeutung $[5,6]$. Dies gilt insbesondere angesichts des Verhaltens vieler Menschen, die einen großen Teil ihrer Freizeit im Freien und möglichst in der Sonne verbringen.

\section{Methoden}

Die Messungen der ultravioletten Sonnenstrahlung wurden an einem heiteren, wolkenlosen Sommertag wenige Tage nach der Sommersonnenwende in Westerland/Sylt auf der geographischen Position $54^{\circ} 55,5^{`} \mathrm{~N}, 8^{\circ} 18,6^{`} \mathrm{O}$ durchgeführt. Die Deklination der Sonne betrug $23,3^{\circ}$, so dass eine Mittagshöhe der Sonne von $58,4^{\circ}$ erreicht wurde. Die Lage des Institutsgebäudes auf dem westlichen Dünenwall der Nordseeinsel Sylt erlaubt Messungen der Sonnenstrahlung bei völlig freiem Horizont ohne jede Abschattung durch Erhebungen, Gebäude oder Vegetation.
Die Eingangsoptik des Radiometers, ein angenähert cosinusgetreuer Teflondiffusor, war auf dem Dach in einer schwenkbaren Halterung montiert. Der Empfänger war entweder horizontal oder direkt auf die Sonne ausgerichtet. In der horizontalen Position wurde der Diffusor durch eine Scheibe von $35 \mathrm{~mm}$ Durchmesser verschattet, die in $40 \mathrm{~cm}$ Entfernung positioniert war. In dieser Konstellation wurden die direkten Sonnenstrahlen ausgeblendet und nur die diffus gestreute Strahlung gemessen. War der Diffusor direkt auf die Sonne gerichtet, wurde umgekehrt die indirekte Sonnenstrahlung mit einer rohrförmigen $40 \mathrm{~cm}$ langen Blende abgeschirmt, so dass nur die direkte Sonnenstrahlung erfasst wurde. Die Blende hatte einen Öffnungshalbwinkel von $2,5^{\circ}$ und einen Böschungswinkel von jeweils $1^{\circ}$ und entsprach damit den Richtlinien der Weltmeteorologenorganisation für die Messung der direkten Sonnenstrahlung mit Pyrheliometern [7]. Die in dieser Anordnung gemessene direkte Strahlung wurde unter Berücksichtigung der Sonnenhöhe auf eine horizontale Fläche umgerechnet, so dass für den Vergleich der Anteile sowohl die indirekt-diffuse als auch die direkte Sonnenstrahlung einheitlich auf die horizontale Fläche bezogen sind.

Vom Diffusor wurde das Licht über einen Quarzlichtwellenleiter in den Eingangsspalt des Radiometers übertragen. Der Spektralapparat bestand aus einem Doppelmonochromator, d.h. zwei in Serie angeordneten Monochromatoren mit jeweils 150 mm Brennweite (DM 150, Bentham Instruments, Reading, England), in welchen das Licht durch holographische Beugungsgitter mit 2400 Linien pro mm spektral zerlegt wurde. Die nominelle Bandbreite des Doppelmonochromators betrug 1,3 nm. Zur Einstellung der jeweiligen Wellenlänge wurden die beiden über einen Spindeltrieb mechanisch gekoppelten Gitter durch einen Schrittmotor positioniert. Am Ausgangsspalt des Spektrometers wurde die monochromatische Strahlung von einem Photonen-Elektronen-Vervielfacher in ein elektrisches Signal gewandelt, welches über einen programmgesteuerten Stromverstärker einem Analog-Digital-Wandler zugeführt wurde. Die Steuerung des Schrittmotors, Schaltung des Stromverstärkers, Datenaufnahme und Speicherung erfolgten programmgesteuert durch einen Personal-Computer.

Die Wellenlängenjustage des Mess-Systems wurde mit einer Niederdruck-Quecksilber-Spektrallinienlampe (Pen Ray Quecksilber/Argon, L.O.T.-Oriel GmbH, Darmstadt) vorgenommen. Die Wellenlängengenauigkeit betrug $\pm 0,15 \mathrm{~nm}$ über den Bereich von $290 \mathrm{~nm}$ bis $450 \mathrm{~nm}$. Die Systemempfindlichkeit wurde mit einem $1000 \mathrm{~W}$ Halogenstrahler kalibriert, einem Sekundärnormal, welches von einem von der PhysikalischTechnischen-Bundesanstalt Braunschweig geeichten Halogenstrahler abgeleitet war.

Die Messungen der direkten und der diffusen Strahlung wurden im Tagesverlauf alternierend durchgeführt jeweils in Intervallen, die ungefähr $5^{\circ}$ Sonnenhöhendifferenz entsprachen.

\section{Ergebnisse}

In Abb. 1 sind die Bestrahlungsstärken für ausgewählte Wellenlängen des UV-Spektrums in Abhängigkeit vom Sonnenhöhenwinkel wiedergegeben. Die beiden Diagramme der oberen Reihe zeigen das Verhalten der Wellenlängen $305 \mathrm{~nm}$ und $310 \mathrm{~nm}$ aus dem UVB-Spektralbereich, die vier Diagramme in den unteren beiden Reihen die Verhältnisse für die UVA-Wel- 

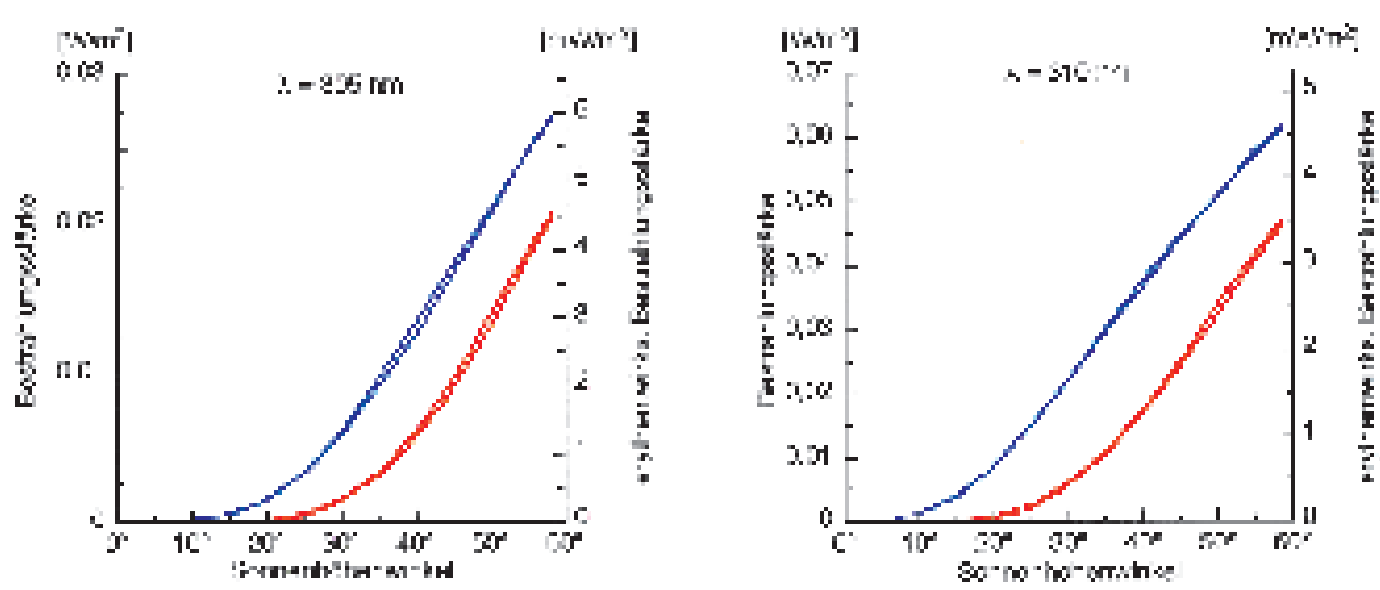

Abb. 1 Die Bestrahlungsstärken der direkten (rote Kurven) und indirekt diffus gestreuten (blaue Kurven) Sonnenstrahlung von verschiedenen Wellenlängen des ultravioletten Spektrums in Abhängigkeit vom Sonnenhöhenwinkel. Die linken Ordinaten geben jeweils die physikalische Bestrahlungsstärke wieder, die rechten Ordinaten die nach dem CIE-Wirkungsspektrum für das Erythem
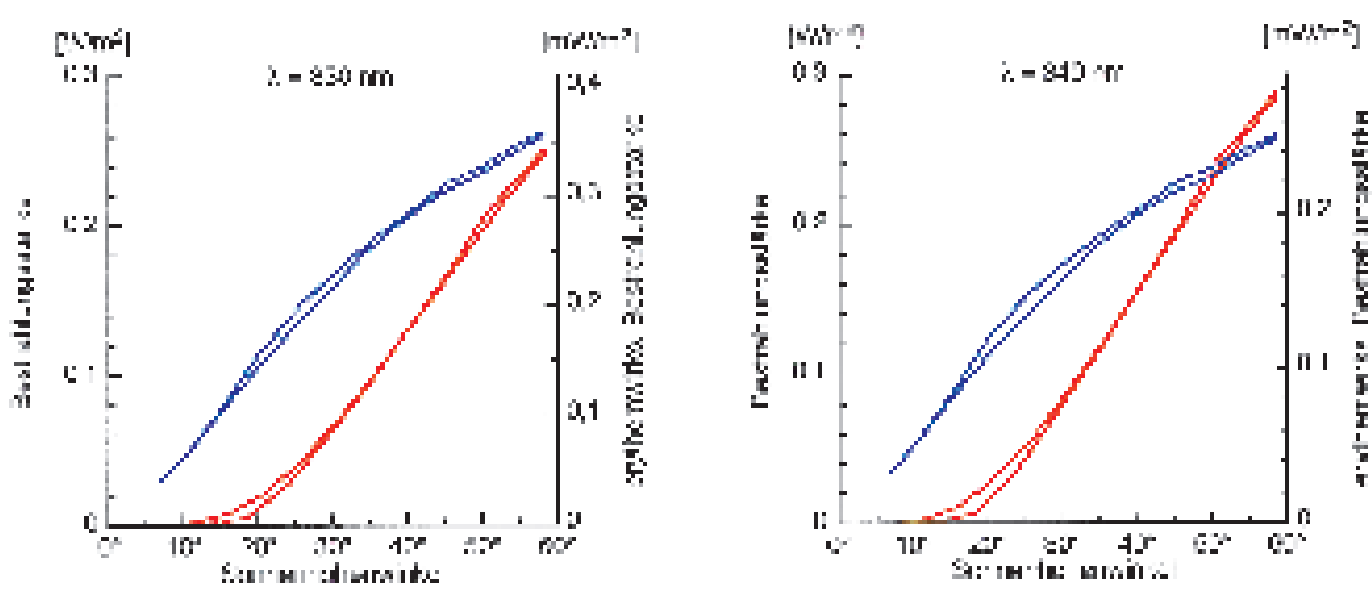
gewichtete Bestrahlungsstärke.
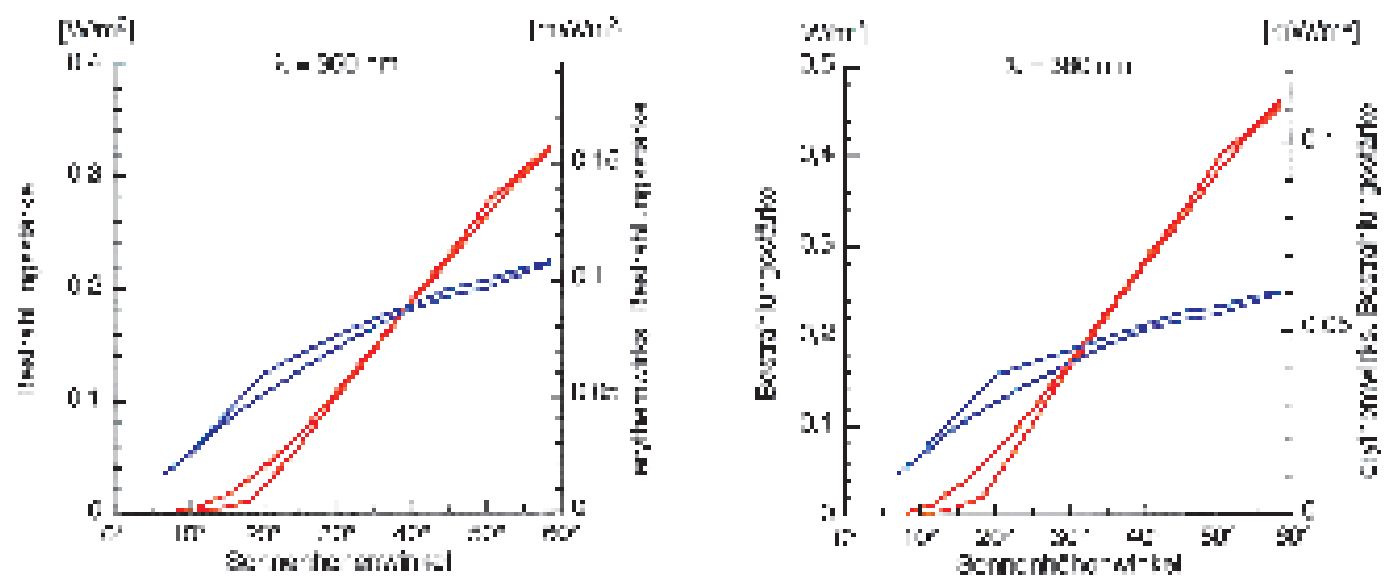

lenlängen 330, 340, 360 und $380 \mathrm{~nm}$. Um die Verläufe vergleichen zu können, sind die Ordinaten-Maßstäbe jeweils so gewählt, dass die Kurven ungeachtet der tatsächlich sehr unterschiedlichen Bestrahlungsstärken, mit denen die Wellenlängen im Sonnenspektrum vertreten sind, ähnlich groß erscheinen. Zusätzlich zur physikalischen Bestrahlungsstärke auf den linken Ordinaten berücksichtigen die Skalierungen der rechten y-Achsen die Erythemwirksamkeit der Wellenlängen entsprechend dem genormten CIE-Wirkungsspektrum für das Erythem $[1,8]$. Diese Skalierungen lassen erkennen, dass die Wellenlänge mit der geringsten physikalischen Bestrahlungs- stärke, nämlich $305 \mathrm{~nm}$, nach der biologischen Wichtung die höchste erythemwirksame Bestrahlungsstärke aufweist und umgekehrt $380 \mathrm{~nm}$ mit den höchsten physikalischen Werten die geringste biologische Wirkung entfaltet. Dazwischen finden sich alle Übergänge.

In den Diagrammen sind jeweils zwei Kurvenzüge abgebildet, die blauen Kurven zeigen die diffus gestreute Strahlung, die roten geben jeweils die direkte Sonnenstrahlung an. Bereits auf den ersten Blick sind sowohl in den Kurvenformen als auch in den Verhältnissen zwischen diffuser und direkter Strahlung er- 
hebliche Unterschiede zu erkennen. Die beiden Wellenlängen aus dem UVB-Spektralbereich zeigen allerdings untereinander ziemliche Ähnlichkeiten: Die Verläufe sind bei geringen Sonnenhöhenwinkeln zunächst sehr flach, die Bestrahlungsstärken äußerst gering. Mit steigender Sonnenhöhe nehmen die Bestrahlungsstärken dann aber überproportional steil zu und zeigen selbst bis zum Sonnenhöchststand von $58,4^{\circ}$ kaum Tendenzen zur Abflachung. Bei beiden Wellenlängen ist die indirekt diffuse Strahlung über den gesamten Bereich erheblich größer als die direkte Strahlung. Insbesondere nimmt die diffuse Strahlung bereits mit geringeren Sonnenhöhen stärker zu als die direkte. Die Unterschiede zwischen den beiden Wellenlängen sind eher diskret: So sind die Übergänge in die steilen Abschnitte der Kurven bei $305 \mathrm{~nm}$ zu höheren Winkeln verschoben. Die Bestrahlungsstärken bei $305 \mathrm{~nm}$ nehmen also erst bei noch höherem Sonnenstand zu als die bei $310 \mathrm{~nm}$.

Im Vergleich hierzu werden im UVA-Spektralbereich bereits bei $330 \mathrm{~nm}$ deutliche Unterschiede manifest: Während die direkte Sonnenstrahlung noch ein weitgehend ähnliches Bild wie im UVB-Bereich in Abhängigkeit von der Sonnenhöhe aufweist, zeigt die diffuse Strahlung einen zur Abszisse konkaven Verlauf. Bei geringen Sonnenhöhenwinkeln ist die diffuse Strahlung zwar schon vergleichsweise stark, mit zunehmender Sonnenhöhe flacht die Kurve jedoch immer mehr ab. Bei Sonnenhöchststand sind diffuse und direkte Strahlung fast gleich groß.

Diese Tendenz wird zu den längeren Wellenlängen immer deutlicher. Die Kurven der diffusen Strahlung flachen mit zunehmender Wellenlänge immer stärker und bereits bei geringeren Sonnenhöhenwinkeln ab: Bei $340 \mathrm{~nm}$ wird das Verhältnis $1: 1$ zwischen diffuser und direkter Strahlung etwa bei der Sonnenhöhe von $50^{\circ}$ erreicht, bei $360 \mathrm{~nm}$ schon bei ungefähr $40^{\circ}$ und bei $380^{\circ}$ schließlich bei rund $30^{\circ}$. Dabei werden die Steigerungen der diffusen Strahlung mit größeren Sonnenhöhen immer geringer, während die direkte Strahlung bei allen Wellenlängen bis zum Sonnenhöchststand weitgehend ungebrochen zunimmt. Dies bedeutet, dass der Anteil der diffusen Strahlung mit zunehmender Wellenlänge und höherem Sonnenstand immer geringer wird.

Bei einer Sonnenhöhe von $30^{\circ}$ beträgt der Anteil der diffusen Strahlung an der Globalstrahlung bei den beiden Wellenlängen $305 \mathrm{~nm}$ und $310 \mathrm{~nm}$ rund $80 \%$, bei $340 \mathrm{~nm} 66 \%$ und bei $380 \mathrm{~nm}$ noch etwas mehr als die Hälfte. Werden die Werte auf den Sonnenhöhenwinkel von $60^{\circ}$ extrapoliert, so zeigt sich, dass im UVB-Bereich bei $305 \mathrm{~nm}$ und $310 \mathrm{~nm}$ der diffuse Anteil mit etwa 56\% noch mehr als die Hälfte der Globalstrahlung ausmacht. Bei dieser Sonnenhöhe werden die Anteile der diffusen Strahlung im UVA-Bereich zu längeren Wellenlängen deutlich geringer. Bei $340 \mathrm{~nm}$ sinkt der Anteil der indirekten Strahlung bereits auf weniger als die Hälfte (46\%) und bei $380 \mathrm{~nm}$ beträgt die diffuse Strahlung nur noch etwa ein Drittel der Globalstrahlung.

Den Diagrammen der Abb. 1 ist außerdem zu entnehmen, dass der Tagesgang der Strahlung an klaren Tagen gänzlich von der Sonnenhöhe bestimmt wird. Die am Vor- und Nachmittag gemessenen Werte liegen fast durchgehend auf identischen Kurven. Eine Abweichung hiervon zeigte sich nur, als die Sonne am Nachmittag unter $20^{\circ}$ gesunken war. Hier nahm die direkte
Strahlung gegenüber dem Vormittag etwas ab, die diffuse Strahlung dagegen zu.

Zusammenfassend ergibt die Darstellung der spektralen Messungen, dass die kürzesten Wellenlängen des ultravioletten Sonnenspektrums auf den geographischen Breiten Deutschlands selbst bei mittäglichen Sonnenhöchstständen im Sommer zum größeren Teil als diffus gestreute Strahlung am Boden eintreffen, während der Beitrag direkter Strahlung geringer ist. Mit zunehmender Wellenlänge nimmt der Anteil der diffusen Strahlung im UVA-Bereich ständig ab.

\section{Diskussion}

Die Sonnenstrahlung wird bei ihrem Durchtritt durch die Atmosphäre von verschiedenen Prozessen beeinflusst und in Abhängigkeit von der Wellenlänge geschwächt. Die im Zusammenhang dieser Arbeit interessierenden Effekte resultieren ihrer physikalischen Natur nach aus der Absorption und Streuung der Strahlung.

Für die Absorption im Bereich der Ultraviolettstrahlung ist das Ozon, das sich zu etwa $90 \%$ in der Stratosphäre-als sog. Ozonschicht - befindet, entscheidend. Der spektrale Absorptionskoeffizient des Ozons nimmt im UVB-Band äußerst steil zu, in den Grenzen des UVB von $315 \mathrm{~nm}$ bis $280 \mathrm{~nm}$ etwa um den Faktor 80, bis zum Maximum der Ozon-Absorptionsbande bei $255,5 \mathrm{~nm}$ sogar um das $237 \mathrm{fache}$. Zwischen den beiden in Abb. 1 gezeigten Wellenlängen $310 \mathrm{~nm}$ und $305 \mathrm{~nm}$ nimmt das spektrale Absorptionsvermögen des Ozons noch um das Doppelte zu [9]. Da die Schwächung durch Absorption dem exponentiellen Lambert-Beerschen Gesetz gehorcht, ist sie extrem von der optischen Weglänge im schwächenden Medium abhängig. Im Falle der Sonnenstrahlung ist die optische Weglänge durch die Atmosphäre von der Sonnenhöhe bestimmt. Im Vergleich zum senkrechten Durchtritt verlängert sich der Weg annähernd um den Faktor $1 / \sin \gamma$, d.h. beispielsweise, dass die optische Weglänge bei einem Sonnenhöhenwinkel von $\gamma=30^{\circ}$ um den Faktor 2 gegenüber dem senkrechten Durchtritt durch die Atmosphäre verlängert ist. Bei der Sonnenhöhe von $60^{\circ}$ beträgt die Zunahme der Weglänge dagegen lediglich das 1,15fache. Aus diesen Gegebenheiten erklärt sich ganz wesentlich die ungewöhnlich starke Abhängigkeit der UVB-Strahlung von der Sonnenhöhe, wie sie für die Wellenlängen $305 \mathrm{~nm}$ und $310 \mathrm{~nm}$ aus der Abb. 1 zu entnehmen ist. Da im UVA keine entsprechend starke Absorption stattfindet, ist die Abhängigkeit der Bestrahlungsstärke von der Sonnenhöhe in diesem Spektralbereich wesentlich geringer ausgeprägt. Die praktische Bedeutung der enorm starken Abhängigkeit der kurzwelligen Ultraviolettstrahlung vom Sonnenhöhenwinkel liegt in der großen Sonnenbrandgefahr bei hohen Sonnenständen, also um die Mittagszeit, im Sommer und auf geringen geographischen Breiten [10].

Bei der Streuung, dem zweiten physikalischen Prozess, der zur Schwächung der Strahlung in der Atmosphäre führt, sind zwei Fälle zu unterscheiden: Sind die Teilchen, an denen die Strahlung gestreut wird, größer oder zumindest von ähnlicher Größe wie die Wellenlänge der Strahlung, so kommt es zur sogenannten Mie-Streuung [11,12]. Diese Art der Streuung findet an Aerosol-Partikeln und an Wassertröpfchen sowie Eiskristallen der Wolken statt. Die direkte Strahlung wird durch die vielfache Beugung an diesen Teilchen in verschiedene Richtungen 
gelenkt. Danach erreicht ein Teil den Boden als diffuse Strahlung. Ein anderer je nach Aerosolgehalt und Bewölkung unterschiedlich großer Teil der Strahlung wird reflektiert. Das Ausmaß dieser Streuung hängt nur sehr wenig von der Wellenlänge der Strahlung ab, so dass ein dunstiger Himmel mit hoher Aerosolkonzentration weiß, ein bewölkter Himmel grau erscheint. Bei durch Wolken verdeckter Sonne ist naturgemäß die gesamte aus dem oberen Halbraum einfallende Strahlung diffus.

Ist die Luft sehr trocken und enthält weder Wassertropfen noch größere Aerosole, so wird die dann tiefblaue Farbe des Himmels von der zweiten Art der Streuung bestimmt. Bei dieser sog. Rayleigh-Streuung $[12,13]$ wird die Strahlung an Teilchen gestreut, deren Durchmesser sehr viel kleiner als die Wellenlänge der Strahlung ist. Im Falle der Sonnenstrahlung sind dies die Luftmoleküle der Atmosphäre. Sie werden zu Schwingungen angeregt und senden dadurch die eingestrahlte Energie wieder in verschiedene Richtungen aus. Dies hat zwei Konsequenzen: Einerseits wird ein Teil der einfallenden Sonnenstrahlung zurück in den Weltraum gestreut, wodurch die direkte Sonnenstrahlung an der Erdoberfläche geschwächt ist. Andererseits erreicht ein Teil der Sonnenstrahlung die Erde auch bei klarem wolkenlosen Himmel nicht direkt, sondern als diffuse Strahlung. Nach vielfacher Streuung an den Luftmolekülen der Atmosphäre strahlt sie diffus aus allen Richtungen des Himmels ein, also auch in den Schatten, der von direkten Sonnenstrahlen nicht beschienen wird.

Entscheidend für die Interpretation der in dieser Arbeit dargestellten Messergebnisse ist die Tatsache, dass die RayleighStreuung äußerst stark von der Wellenlänge der Strahlung abhängt. Sie verhält sich umgekehrt proportional zur 4. Potenz der Wellenlänge. Das bedeutet, dass die kurzwellige Strahlung erheblich stärker gestreut wird als die längerwelligere.

Auch die Streuung hängt wie die Absorption in exponentieller Weise von der optischen Weglänge durch die Atmosphäre ab. Entsprechend ist die Streuung bei niedrigem Sonnenstand stärker als bei hohem. Diese Sachverhalte finden sich in den in Abb. 1 gezeigten Messungen von direkter und indirekt gestreuter Strahlung wieder: Bei den Wellenlängen $305 \mathrm{~nm}$ und $310 \mathrm{~nm}$ übertrifft die Stärke der indirekten Strahlung die der direkten selbst bei der mittäglichen Sonnenhöhe von 58,4 . Die Kurven liegen so weit auseinander, dass es erlaubt erscheint, diese Aussage auch für ganz Deutschland zu generalisieren, also auch auf südlichere Orte wie München oder Freiburg zu übertragen, bei denen die sommerlichen Mittagshöhen der Sonne entsprechend der geographischen Breite von $=48^{\circ} \mathrm{N}$ etwa $65^{\circ}$ erreichen. Dies bedeutet, dass die biologisch besonders wirksamen Wellenlängen im UVB während des gesamten Tages zum größeren Teil diffus aus dem Himmelsgewölbe eingestrahlt werden.

Im UVA-Bereich lässt sich eindrucksvoll erkennen, wie sich die Verhältnisse von direkter und diffuser Strahlung mit der Wellenlänge ändern. Im Bereich von $\mathrm{UVA}_{2}$ bei $330 \mathrm{~nm}$ und $340 \mathrm{~nm}$ sind die Stärken direkter und diffuser Strahlung bei sommerlich-mittäglichen Sonnenhöhen etwa gleich, bei längeren Wellenlängen im $\mathrm{UVA}_{1}$-Bereich rückt der Punkt, an dem direkte und indirekte Strahlung gleich viel zur Globalstrahlung beitragen, zu immer geringeren Sonnenhöhen. Bei der Wellenlänge $380 \mathrm{~nm}$ am Übergang zum sichtbaren Licht nimmt die indirekt diffuse Strahlung bei Sonnenhöhen jenseits von $30^{\circ}$ nur noch verhältnismäßig wenig zu. Dagegen steigt die direkte Strahlung auch bei dieser Wellenlänge bis zur maximalen Sonnenhöhe weiter an.

Insgesamt lässt sich feststellen, dass sich die ultraviolette Sonnenstrahlung nicht allein in Hinblick auf ihre spektrale Zusammensetzung in Abhängigkeit von der Sonnenhöhe ständig im Tages- und Jahresverlauf ändert [4], sondern dass sich dabei auch die Verhältnisse von direkter und indirekt diffuser Strahlung ständig verschieben. Die kürzesten Wellenlängen im UVB-Spektralbereich, also zugleich die Wellenlängen, welche an der Haut die größten Wirkungen entfalten $[1,8]$, sind am stärksten der Streuung unterworfen und zeichnen sich selbst bei den höchsten Sonnenständen, die auf den Breiten Deutschlands vorkommen, dadurch aus, dass der größere Teil als indirekt-diffuse Strahlung am Erdboden eintrifft.

Inwieweit lassen sich die mitgeteilten Ergebnisse verallgemeinern? Wie erwähnt hängt der diffuse Anteil für jede Art Sonnenstrahlung stark davon ab, wie transparent oder trüb die Atmosphäre ist. Der diffuse Anteil für die gesamte Sonnenstrahlung betrug um die Mittagszeit des Tages, an dem die Messungen durchgeführt wurden, knapp $12 \%$ der Globalstrahlung. Dies ist ein für heitere klare Tage typischer Wert [14]. Lediglich am späten Nachmittag kam es im Westen zu einer vermehrten Trübung des Himmels in Horizontnähe. Dies äußerte sich im UVA- Bereich darin, dass die direkte Strahlung ab-, die indirekt diffuse Strahlung dagegen zunahm (vgl. Abb.1, zweite und dritte Reihe: $330 \mathrm{~nm}$ bis $380 \mathrm{~nm}$ ).

Messfehler dürften die Ergebnisse lediglich durch die nicht ideale Kosinustreue des verwendeten Diffusors beeinflusst haben [15]. Jedoch zeigte ein stichprobenartiger Vergleich mit einem zwischenzeitlich zur Verfügung stehenden, wesentlich verbesserten Diffusor lediglich Abweichungen von wenigen Prozentpunkten. Da die Messungen der direkten und der indirekten UV-Strahlung jeweils unmittelbar nacheinander mit demselben System durchgeführt wurden, haben Fehler in der Kalibrierung, die sich auf etwa $\pm 5 \%$ addieren können [16], lediglich Einfluss auf die Absolutwerte, nicht jedoch auf die mitgeteilten Relationen von direkter und indirekter Strahlung.

Die gezeigten Verhältnisse erlangen überall dort Bedeutung, wo die Strahlung ohne Abschattung aus dem gesamten Himmelsraum einfallen kann. Dies gilt beispielsweise an der See. Umgekehrt wird der Anteil hautwirksamer Strahlung dort selbst bei direktem Sonneneinfall mehr oder weniger stark gemindert sein, wo größere Teile des Himmels beispielsweise durch Gebäude, Vegetation oder durch die orographischen Gegebenheiten verschattet sind. Das alleinige Ausblenden der direkten Sonnenstrahlen, wie es bei den vorgestellten Messungen erfolgte, führt bei Sonnenhöchststand zu einer Verringerung der UVB-Strahlung auf rund die Hälfte, also zu einem „Sonnenschutzfaktor“ von etwa 2. In der Praxis dürfte die Reduktion der hautwirksamen Strahlung jedoch immer deutlich höher liegen, weil beispielsweise unter einem Sonnenschirm nicht allein die Sonnenscheibe, sondern auch mehr oder weniger große Teile des Himmels aus deren Nachbarschaft verschattet werden. Der Aufenthalt im Schatten wird also im Allgemeinen doch einen größeren Schutz vor Sonnenbrand bieten, als es aus den hier gezeigten Beiträgen von direkter und indirekt diffuser UV-Strahlung abzuleiten ist. Desungeachtet 
zeigen die Messungen jedoch, dass wegen des ungewöhnlich hohen Anteils diffuser Strahlung selbst im Schatten eine nicht zu vernachlässigende UV-Strahlung herrscht. Da sich die kurzwellige UVB-Strahlung mit dieser Eigenschaft vom sichtbaren Licht und der Wärmestrahlung der Sonne grundlegend unterscheidet - wie erwähnt macht die diffuse Strahlung an klaren wolkenlosen Tagen lediglich etwa $10 \%$ bis $15 \%$ der gesamten Sonnenstrahlung aus - ist es völlig unmöglich, die nicht wahrnehmbare UV-Strahlung abzuschätzen.

\section{Literatur}

${ }^{1}$ McKinley AF, Diffey BL. A reference action spectrum for ultraviolet induced erythema in human skin. CIE J 1987; 6: 17-22

2 Krutmann J, Hönigsmann H. Handbuch der dermatologischen Phototherapie und Photodiagnostik. Berlin: Springer, 1997

${ }^{3}$ Altmeyer P, Hoffmann K, Stücker M. Skin cancer and UV radiation. Berlin: Springer, 1997

${ }^{4}$ Stick C, Pielke L. Die Zusammensetzung der solaren UV-Strahlung im Tagesverlauf. Akt Dermatol 1998; 24: 159-163

${ }^{5}$ Phillips NA, Gallagher RP, Coldman AJ, Band PR. Skin cancer incidence: What has happened and what can we expect? In: Volkmer B, Heller H (eds). Environmental UV-radiation. Risk of Cancer and primary prevention. Stuttgart: G. Fischer, 1996: 323 - 332

${ }^{6}$ Strahlenschutzkommission. Schutz des Menschen vor UV-Strahlung, Empfehlungen und Stellungnahmen der Strahlenschutzkommission 1995 - 1997. Bonn: Informationen der SSK des Bundesministeriums für Umwelt, Naturschutz und Reaktorsicherheit, 1998; No. 4

${ }^{7}$ World Meteorological Organisation. Guide to meteorological instruments and methods of observation. Geneva: WMO, 1983; No. 8

${ }^{8}$ Deutsches Institut für Normung e. V, DIN 5031-10. Strahlungsphysik im optischen Bereich und Lichttechnik, Teil 10: Photobiologisch wirksame Strahlung, Größen, Kurzzeichen und Wirkungsspektren. Berlin: Beuth, 2000

${ }^{9}$ Molina LT, Molina MJ. Absolute absorption cross sections of ozone in the 185 to $350 \mathrm{~nm}$ wavelength range. J Geophys Res 1986; 91: $14.501-14.508$

${ }^{10}$ Stick C, Pielke L. Ultraviolette Sonnenstrahlung und Sonnenbrandgefahr. Akt Dermatol 1997; 23: 1 -5

${ }^{11}$ Mie G. Beiträge zur Optik trüber Medien, speziell kolloidaler Metalllösungen. Annalen der Physik, IV. Folge 1908; 25: 377-445

12 Eichler H-J. Interferenz und Beugung. In: Niedrig H (Hrsg). Bergmann Schaefer Lehrbuch der Experimentalphysik. Berlin: Walter de Gruyter, 1993; Band 3 Optik: 301 - 453

${ }^{13}$ Rayleigh JW. On the transmission of light through an atmosphere containing small particles in suspension, and on the origin of the blue of the sky. Phil Mag 1989; 47: 375-384

${ }^{14}$ Deutscher Wetterdienst. Allgemeine Meteorologie. Leitfäden für die Ausbildung im Deutschen Wetterdienst. Offenbach: DWD, 1987; No. 1

${ }^{15}$ Gröbner J, Blumthaler M, Ambach W. Experimental investigation of spectral global irradiance measurement errors due to a non ideal cosine response. Geopys Res Lett 1996; 23.18: 2493-2496

${ }^{16}$ Seckmeyer G, Mayer B, Bernhard G. The 1997 status of solar UV spectroradiometry in Germany: Results from the national intercomparison of UV spectroradiometers Garmisch Partenkirchen, Germany. Schriftenreihe des Frauenhofer-Instituts Atmosphärische Umweltforschung. Aachen: Shaker, 1998; Band 98,55

\section{Prof. Dr. Carsten Stick}

Institut für Medizinische Klimatologie der Universität Kiel Hermann-Rodewald-Straße 5

24098 Kiel

E-mail: c.stick@physiologie.uni-kiel.de 